\title{
Abortos espontáneos en Hospital de Llay-Llay y su relación con labores agrícolas de la madre
}

\author{
JUAN CONTRERAS-LEVICOY(1), ERIKA ASTORGA(1), RODRIGO CASTRO(1), \\ GLADYS YENTZEN(2), MIGUEL CUMSILLE(2)
}

\section{RESUMEN}

Los pesticidas son sustancias quimicas usadas con frecuencia en las zonas agrícolas de nuestro pais. Es un hecho bien establecido que estas sustancias pueden ser dañinas para la salud no sólo del trabajador agricola sino también para su descendencia. Su objetivo es estudiar la asociación entre abortos espontáneos y la ocupación agrícola de la madre. Se efectuó un estudio descriptivo, recopilando las fichas clinicas de aquellas pacientes que presentaron aborto espontáneo registrados en el libro de procedimientos de la maternidad del Hospital San Francisco de Llay-Llay entre enero del 2002 y diciembre del 2003. Se calcula la tasa de incidencia de aborto espontáneo comparándola con las últimas cifras nacionales disponibles, y el porcentaje de ocupación agricola de la madre en relación con la población femenina ocapada de 15 años o más de la misma área geográfica según Censo del 2002. Se realizó la prueba $Z$ para comparación de proporciones, e intervalos de confianza para la razón entre las densidades de incidencia. La tasa de incidencia de aborto espontáneo fue 81,02 casos $/ 1.000$ RNV versus 9,5 casos $/ 1.000 \mathrm{RNV}$ en Chile 1996, otorgando una razón de incidencia de 8,5 veces $(I C=6,72-10,65)$. El porcentaje de ocupación agricola es mayor que la población femenina ocupada de 15 años o más de la misma área geográfica. ( $p<0,0001)$. El riesgo de presentar aborto espontáneo en Llay-Llay y Catemu es mayor que el resto del pais. La ocupación agricola está relacionada con un mayor riesgo de aborto espontáneo, probablemente debida a la exposición a pesticidas.

Palabras clave: Aborto espontáneo, pesticidas, exposición ocupacional.

\begin{abstract}
SPONTANEOUS ABORTIONS IN THE LLAY-LLAY HOSPITAL AND THEIR RELATION TO MOTHER'S AGRICULTURAL LABORS

Pesticides are chemical substances frequently used in agricultural areas of our country. It is a well established fact that these substances can be harmful to the health, not only of agricultural workers, but also to that of their offspring. The objective of this paper is that of studying the relation between spontaneous abortions and the agricultural occupation of the mother. A descriptive study was carried out assembling the clinical records of those patients presenting sponrtaneous abortion registered in the maternity book of procedures of the San Francisco de Llay-Llay Hospital between January 2002 and December 2003. The incidence of spontaneous
\end{abstract}

(1) Interno de Medicina. Facultad de Medicina. Universidad de Chile.jc ancud@yahoo.com

(2) Escuela de Salud Pública. Facultad de Medicina. Universidad de Chile. 
abortion is calculated comparing it to the last available national figures, and the percentage of agricultural occupation of the mother in relation to the 15 year old and over female population of the same geographic area, according to the 2002 Census. The $Z$ test was performed for comparison of proportions and confidence intervals for the ratio between incidence densities. The incidence rate for spontaneous abortion was 81.02 cases/1,000 live-newborn registrations $(R N V)$ versus 9.5 cases/1,000 live-born registrations in Chile 1996, granting an incidence ratio of $8.5(\mathrm{Cl}=6.72-10.65)$. The percentage of agricultural occupation is higher than the female 15 year old and over population of the same geographic area $(p<0.0001)$. The risk of spontaneots abortion occurring in Llay-Llay or Catemu is higher than in the rest of the country. The agricultural occupation is related to a higher risk of spontaneous abortion, probably due to the exposure to pesticides.

Key words: Spontaneous abortion, pesticides, occupational exposure.

\section{INTRODUCCIÓN}

Los pesticidas son productos químicos, naturales o sintéticos utilizados para combatir organismos dañinos en plantaciones de todo el mundo. Se pueden clasificar en insecticidas, herbicidas y fungicidas. Todos son tóxicos en mayor o menor medida.

Hay en uso en Chile, por lo menos, 11 grupos de plaguicidas regulados por el Servicio Agricola Ganadero y prohibida la importación, venta o uso de otros 13; todos potencialmente tóxicos para el ser humano. Chile reconoce la existencia de contaminantes orgánicos persistentes (COPs), los cuales tienen propiedades tóxicas, son resistentes a la degradación, se bioacumulan y son transportados por el aire, el agua y las especies migratorias a través de las fronteras internacionales y depositados lejos del lugar de su liberación, acumulándose en ecosistemas terrestres y acuáticos. Los productos químicos señalados son: aldrin, bifenilos policlorados, clordano, DDT, dieldrina, dioxinas, endrina, furanos, heptacloro, hexaclorobenceno, mirex y toxafeno. Mediante el lnventario Nacional de Existencias de Plaguicidas Caducados (COPs), se compromete a analizar el uso de tales productos y viabilizar su eliminación según lo acordado en el Convenio de Estocolmo sobre COPs!

Los pesticidas son sustancias químicas usadas con frecuencia en las zonas agrícolas de nuestro país. En estas áreas, las actividades de temporada constituyen una importante fuente laboral tanto para hombres como para mujeres. Estas últimas se dedican a la recolección de productos agricolas, quedando expuestas al contacto con pesticidas. Es un hecho bien establecido que estas sustancias pueden ser dañinas para la salud no sólo del trabajador sino también para la descendencia. Se ha descrito asociación con un aumento de la frecuencia de abortos espontáneos $2-4$ y malformaciones congénitas ${ }^{5-7}$ en poblaciones expuestas en estudios extranjeros, considerando como factor de riesgo la exposición del trabajador agrícola a los químicos usados en el tratamiento de las plantaciones. En Chile se realizó un estudio que relacionaba las malformaciones congénitas con la exposición a pesticidas ${ }^{8}$, sin embargo, no existen estadísticas de asociación entre abortos espontáneos y pesticidas.

En las comunas de Llay-Llay y Catemu, ubicadas en la $V$ región de Chile $y$ pertenecientes al Servicio de Salud de Aconcagua, el $20 \%$ de la población femenina ocupada de 15 años o más, se dedica al trabajo agrícola o silvoagricultura (tramo $\mathrm{A}$ ), según datos del censo del año 20029,10. El cual se encuentra constituido, principalmente, por labores de recolección de productos agrícolas, denominado comúnmente como "temporeras".

El objetivo del presente estudio es analizar la incidencia de abortos espontáneos en una zona agrícola y compararlo con la incidencia a nivel nacional; observar si en el grupo de mujeres que presentó aborto espontáneo hay 
mayor proporción de trabajadoras agrícolas en relación con el resto de la población femenina de la misma área geográfica.

Hipótesis: La tasa de incidencia de abortos espontáneos es mayor que la observada a nivel nacional. En el grupo de mujeres que presentó abortos espontáneos, existe una mayor proporción que se dedica a labores agrícolas al ser comparados con el resto de la población femenina de la misma área geográfica.

\section{MATERIAL Y MÉTODO}

Se realizó un estudio descriptivo de los abortos espontáneos registrados en el Hospital San Francisco de Llay-Llay durante el periodo comprendido entre el 1 de enero del 2002 y el 31 de diciembre del 2003. Se consideró como aborto espontáneo aquella pérdida del embarazo que ocurre antes de las 20 semanas, sin que existan maniobras que la induzcan"!.

Los abortos que figuran en el "Libro de Procedimientos" de la sección de Maternidad del Hospital San Francisco de Llay-Llay, corresponden a la población femenina de las comunas de Llay-Llay y Catemu.

Se recolectó el total de fichas clínicas de aquellas pacientes que presentaron diagnóstico de aborto espontáneo registrados en el Libro de Procedimientos de la Maternidad del Hospital San Francisco de Llay-Llay, correspondientes al periodo en estudio, sin incluir los abortos sépticos o provocados. Éste constituyó nuestro universo de estudio. Se registró la edad de la madre, edad gestacional del óbito, y ocupación de la madre.

La mediana de edad de la madre fue de 27 años (16 - 46 años) mientras que la edad gestacional del óbito fue de 12 semanas.

Con el total de abortos, se calcula la tasa de incidencia de aborto espontáneo por cada 1.000 recién nacidos vivos (RNV) y se compara este dato con la última tasa nacional de aborto espontáneo disponible.

Se calculó el porcentaje de ocupación agrícola, y se comparó con el porcentaje de población femenina ocupada mayor de 15 años con actividad agrícola o silvoagricultura (tramo A) de la misma área geográfica según datos del Censo Nacional 2002, 10
Para el análisis estadístico se usó el programa STATA 7.0. Se realizó la prueba $Z$ para comparación de proporciones, e intervalos de confianza para la razón entre las densidades de incidencia.

\section{RESULTADOS}

Se registraron 79 casos de aborto espontáneo correspondientes al periodo en estudio en la población de Llay-Llay y Catemu. En el mismo periodo se registran 975 RNV en la misma área geográfica, resultando una tasa de incidencia de 81,02 casos/1.000 RNV.

En el último registro publicado de egresos por aborto espontáneo del año 1996, bajo el código CIE-IX 634'2 (anuario demografia y egresos), se registraron 2.650 abortos espontáneos y 278.629 RNV corregido, resultado una tasa de incidencia de 9,5 casos/1.000 RNV 13. La razón entre las incidencias es de 8,5 veces, con un intervalo de confianza de $6,72-10,65$ veces.

La ocupación de la madre se obtuvo en el $82,14 \%$ de los casos de aborto espontáneo. De éstas, el 36,09\% se dedicaba al trabajo agrícola, mientras que la ocupación en labores agrícolas o silvoagropecuarias (Tramo A) entre las mujeres mayores de 15 años en las comunas de Llay-Llay y Catemu, es de $20 \%$. Entre ambos grupos se encontró una diferencia de proporciones de $16,51 \%(Z=3,58 ; p<0,0001)$.

\section{DISCUSIÓN}

El riesgo de tener un aborto espontáneo en las comunas en estudio es 8,5 veces mayor que en el resto del país. Concordante con ello, en la V Región de Chile existe un gran desarrollo de la agricultura industrial. El consumo de pesticidas de esta región representa el $31 \%$ del total nacional, y está constituido en un $81 \%$ por fungicidas, bactericidas y herbicidas ${ }^{14}$. Por otro lado, las intoxicaciones agudas por plaguicidas, registradas por la Red Nacional de Vigilancia Epidemiológica en Plaguicidas (Revep) dependiente del Ministerio de Salud, ocurridas en hombres entre los años 1998 y 1999 en el Servicio de Salud Aconcagua, fueron las más altas del país. En los años siguientes 
no continuó siendo la primera, pero el número de casos sigue siendo importante en relación con el resto del país ${ }^{15-17}$.

La población beneficiaria del sistema público en las comunas incluidas en el estudio alcanza el $81,3 \%$, mientras que en el país es del $66,5 \% 18$. Por ello es posible que muchos casos de aborto espontáneo se hayan escapado del registro nacional, y que la incidencia de aborto espontáneo en el país sea mayor a la calculada en el presente estudio, lo que se traduciría en un riesgo de aborto espontáneo en éstas comunas menor que las 8,5 veces consideradas inicialmente.

Los resultados sugieren mayor riesgo de aborto espontáneo en las trabajadoras agrícolas. Si bien, no es posible atribuir causalidad, el contacto con pesticidas puede ser uno de los principales factores involucrados, tal como se reporta en otros estudios, en donde se ha visto que no sólo aumenta el riesgo de aborto sino que también las malformaciones congénitas, $y$ distintos tipos de problemas de fertilidad ${ }^{19}$. Las trabajadoras agrícolas tienen otros factores que pudieran explicar la mayor incidencia de abortos, tales como horas trabajadas, educación, estado nutricional, etc, datos que no fueron tabulados 20 etc. En otros estudios estas dificultades fueron soslayadas mediante el estudio del padre trabajador agricola, considerando una exposición indirecta de la madre, a través de la ropa del trabajador o herramientas de trabajo ${ }^{20}$. Sin embargo, esta metodología muestra menor asociación de malformaciones congénitas con pesticidas, excepto cuando el padre los manipula ${ }^{22}$, mientras que en otras no muestra asociación ${ }^{23}$. En nuestro caso, dado que el registro de la ocupación paterna generalmente estaba ausente, no fue posible considerarlo en el estudio.

La incidencia de aborto espontáneo observada en nuestro estudio es cercana al doble de la incidencia de malformaciones congénitas reportada por A. Rojas, quien incluyó en su estudio a los $R N$ vivos $y$ mortinatos portadores de malformación del Hospital de Rancagua ${ }^{8}$. Lo anterior puede explicarse por el hecho de que un número cada vezmenor de embriones o fetos lograran etapas más avanzadas de desarrollo, naciendo sólo aquellos que no tuvieron daños letales. En otras palabras, el daño ocasionado por los pesticidas afecta desde etapas precoces del desarrollo embrionario, causando la pérdida aproximada de dos fetos por cada recién nacido malformado. Apoyando lo anterior, en otro estudio de incidencia de malformaciones congénitas se observó que la incidencia máxima de muerte fetal por anomalias congénitas ocurría cuando éstos eran concebidos durante el periodo de mayor uso de pesticidas lo cual sugiere un efecto dosis dependiente 21 .

El estudio de aborto espontáneo permite obviar las dificultades que ofrece el diagnóstico de las malformaciones congénitas, debido principalmente a su gran heterogeneidad, que incluye desde malformaciones menores de la piel hasta malformaciones del sistema nervioso central.

La maternidad del hospital de Llay-Llay tiene asignada la población de un área específica (Llay-Llay y Catemu) y los procedimientos de legrado y revisión post-aborto no son derivados, lo cual hizo más confiable el cálculo de incidencia.

En estudios que han buscado la efectividad con que los trabajadores agrícolas usan las medidas de protección personal contra los pesticidas se ha encontrado que se utilizan muy deficientemente ${ }^{24}$.

De acuerdo al resultado, se hace necesario incrementar las actuales medidas destinadas a proteger al trabajador agricola. Son necesarios estudios posteriores que investiguen esta asociación y que permitan atribuir, con mayor grado de certeza causalidad a los pesticidas, en sus distintas familias, en relación con el aborto espontáneo.

\section{REFERENCIAS}

1. CHILE. SERVICIO AGRICOLA GANADERO. (2003). Inventario Nacional de Existencias de Plaguicidas Caducados (COPS). [en linea]. Disponible: http://www.sag.gob.cl>. [Consulta: 2004, Agosto 91.

2.- RODRIGUEZ C. Toxicología laboral en la reproducción y el desarrollo. Salud ocup. (Buenos Aires); nov.dic. 2000; 18(78): 12-20.

3.- ARBUCKLE TE, SEVER L E. Pesticide exposures and fetal death: a review of the epidemiologic literature. Crit Rev Toxicol 1998; $28(3): 229-70$. 
4.- NURMINEN T. Maternal pesticide exposure and pregnancy outcome. J Occup Environ Med 1995; 37(8): 935-40.

5.- MEDINA-CARRILO L, RIVAS-SOLIS F, FERNÁNDEZ-ARGUELLES R. Risk for congenital malformations in pregnant women exposed to pesticides in the state of Nayarit, Mexico. Ginecol Obstet Mex 2002; 70: 538-44.

6.- BELL, E M, HERTZ-PICCIOTTO I, BEAUMONT J J. Case-Cohort Analysis of Agricultural Pesticide Applications near Maternal Residence and Selected Causes of Fetal Death. Am J Epidemiol 2001; 154 : $702-10$.

7.- KRISTENSEN P, IRGENS $1, M$, ANDERSEN A, BYE A S, SUNDHEIM L. Birth defects among offspring of Norwegian farmers, 1967-1991. Epidemiology 1997; 8(5): 537-44

8.- ROJAS A, OJEDA $M$ E, BARRAZA $X$. Malformaciones congénitas y exposición a pesticidas. Rev Méd Chile 2000; 128(4): 399-404.

9.- CHILE. INSTITUTO NACIONAL DE ESTADÍSTICAS. Resultados Generales Censo 2002. Población de 15 años o más ocupada, por rama de actividad económica, según área urbana-rural, sexo y años de estudio aprobados. Comuna de Catemu. [en línea]. Disponible: <http://www.ine.cl/cd2002/ cuadros/9/C9A_05702.pdf $>$ [Consulta: 2004, Julio 23].

10.- CHILE. INSTITUTO NACIONAL DE ESTADÍSTICAS. Resultados Generales Censo 2002. Población de 15 años o más ocupada, por rama de actividad económica, según área urbana-rural, sexo y años de estudio aprobados. Comuna de LlayLlay.[en linea]. Disponible: $\angle$ http://www.ine.cl/ cd2002/cuadros/9/C9A_05703.pdf > [Consulta: 2004, Julio 23].

11.- PÉREZ SÁNCHEZ, DONOSO SIÑA. Obstetricia. Editorial Mediterráneo, Segunda Edición. Santiago 1992. pp 455-73.

12. - CHILE. INSTITUTO NACIONAL DE ESTADÍSTICAS. Amuario de Demografia 1996. Santiago: INE, 1998.

13.- CHILE. MINISTERIO DE SALUD. Anuario de egresos hospitalarios año 1996. Santiago: Ministerio de Salud, 1996.

14.- CHILE. Servicio Agrícola y Ganadero (SAG). Santiago, 2004. [Resumen por región de los Plaguicidas Agrícolas vendidos en el pais (año
1999)] datos aportados al autor por Subdepartamento Plaguicidas y Fertilizantes.

15.- CHILE. MINISTERIO DE SALUD, DEPARTAMENTO DE EPIDEMIOLOGIA. Intoxicaciones agudas por plaguicidas según servicio de salud $y$ causa de intoxicación. Chile, enero a junio $200 \mathrm{l}$. Boletín e-vigfa $N^{\circ} 1$. [en linea]. Disponible: <http: /epi.minsal.cl/evigant/Numero01/pag43.htm> [2004, Julio 23]

16.- CHILE. MINISTERIO DE SALUD, DEPARTAMENTO DE EPIDEMIOLOGÍA. Intoxicaciones por Plaguicidas. Año 2004. El Vigía. Número: 25. [en línea]. Disponible: <http://epi.minsal.cl/evigant/ Numero25/evigia/index.htm> [2004, Julio 23].

17.- CHILE. MINISTERIO DE SALUD, DEPARTAMENTO DE EPIDEMIOL.OGÍA. Intoxicaciones por Plaguicidas, enero - junio de 2004. El Vigía. Número: 26 [en línea]. Disponible: <http:// epi.minsal.cl/evigant/Numero26/evigia/index.htm>. [2004, Julio 23].

18.- CHILE. MINISTERIO DE PLANIFICACIÓN. Población según sistema previsional de salud: LlayLlay y Catemu. [en línea]. Disponible: <http:// www.mideplan.c]>. [2004, Diciembre 12]

19.- SUNIL KUMAR. Occupational Exposure Associated with Reproductive Dysfunction. J Occup Health 2004; 46: 1-19.

20. FAUNDES A, HARDY E, DIAZ J, PINOTTI J. Association of marital status and years of schooling with perinatal outcome: the influence of pre-natal care as an intermediate variable. J Perinat Med 1982; 10(2): 105-13.

21.- REGIDOR E, RONDA E, GARCÍA A M, DOMÍNGUEZ, V. Paternal exposure to agricultural pesticides and cause specific fetal death. Occup Environ Med 2004; 61(4): 334-9.

22. - GARCIA A M, FLETCHER T, BENAVIDES F G, ORTS E. Parental agricultural work and selected congenital malformations. Am J Epidemiol 1999; 149(1): 64-74

23.- ROAN C C, MATANOSKI GE, MCILNAY C Q, OLDS K L, PYLANT F, TROUT J R et al. Spontaneous abortions, stillbirths, and birth defects in families of agricultural pilots. Arch Environ Health 1984; 39(1): 56-60.

24. GARCÍA A M, RAMíREZ A, LACASAÑA $M$. Prácticas de utilización de plaguicidas en agricultores. Gac Sanit 2002; 16(3): 236-40.

Usted puede comentar éste y otros artículos publicados en la Revista Chilena de Salud Pública, enviando un correo electrónico a revistasp(amed.uchile.cl 